\title{
An Interesting Case of Button Battery causing Septal Perforation
}

\author{
${ }^{1}$ Sonia Seth, ${ }^{2}$ Harvinder Kumar
}

\begin{abstract}
Button batteries presenting as foreign bodies in the nasal cavity are rarely seen but common in pediatric age group. They are hazardous due to the extensive nature of damage caused by them. In this study, we discuss the case of a child with impacted button battery in the nasal cavity. A 7-year-old boy presented with bilateral foul-smelling nasal discharge since 6 months. It was purulent, yellowish-green, often blood stained. Radiograph showed evidence of a button battery in the left nostril. The foreign body was removed endoscopically and it was found that there was a perforation in the nasal septum. Early recognition and management is the key to prevention of complications due to foreign bodies, especially corrosive type.
\end{abstract}

Keywords: Button battery, Foreign body nose, Septal perforation.

How to cite this article: Seth S, Kumar H. An Interesting Case of Button Battery causing Septal Perforation. Clin Rhinol An Int J 2017;10(1):22-24.

\section{Source of support: Nil}

\section{Conflict of interest: None}

\section{INTRODUCTION}

Button batteries are increasingly being used in many electrical devices like toys, watches, calculators, etc. They are composed of alkaline contents. Due to their small size, these batteries can be very easily inserted by small children in the nose. Button battery found as a foreign body in the nasal cavity is rare, and it is most commonly discovered in children between the ages of 2 and 5 years. ${ }^{1}$ A nasal button battery is an otorhinolaryngological emergency. Button batteries can lead to severe damage, necrosis, and perforation of the nasal septum, intranasal synechiae, and nasal deformity. ${ }^{2}$ Button batteries should ideally be removed by an otorhinolaryngologist in a controlled setting under general anesthesia if they cannot be removed in the outpatient department. We hereby report this case to advocate that button battery in the nasal cavity

\footnotetext{
${ }^{1}$ Professor, ${ }^{2}$ Professor and Head

1,2Department of ENT, Punjab Institute of Medical Sciences Jalandhar, Punjab, India

Corresponding Author: Sonia Seth, Professor, Department of ENT, Punjab Institute of Medical Sciences, Jalandhar, Punjab India, Phone: +919463326264, e-mail: drsoniaaroraent@gmail. com
}

is a serious condition and early diagnosis and management is the key to prevention of grave complications like septal perforation, nasal synechiae, etc.

\section{CASE REPORT}

A 7-year-old male child presented to the Department of Otorhinolaryngology, Punjab Institute of Medical Sciences, Jalandhar, Punjab, India, with foul-smelling bilateral nasal discharge since 6 months. The discharge was purulent, yellowish, and often blood stained. There was no history of foreign body insertion given by the parents. On anterior rhinoscopy examination, there was yellowish-green mucopurulent, foul-smelling discharge seen in both the nasal cavities. Radiograph of nose and paranasal sinuses (Water's view and lateral view) showed a small round metallic object in the nostril (Figs 1 and 2).

The child was taken up for endoscopic removal of the foreign body under general anesthesia. Preoperative routine investigations like complete blood count, bleeding time, clotting time, urine $\mathrm{C} / \mathrm{E}$, Random blood sugar, blood urea, serum creatinine, $\mathrm{X}$-ray chest posteroanterior view were done. On further evaluation under endoscopic guidance, nasal cavity on both sides was filled with mucopurulent yellowish-green discharge which was cleared with suction, and a perforation in the nasal septum was revealed. The foreign body was impacted in the septum.

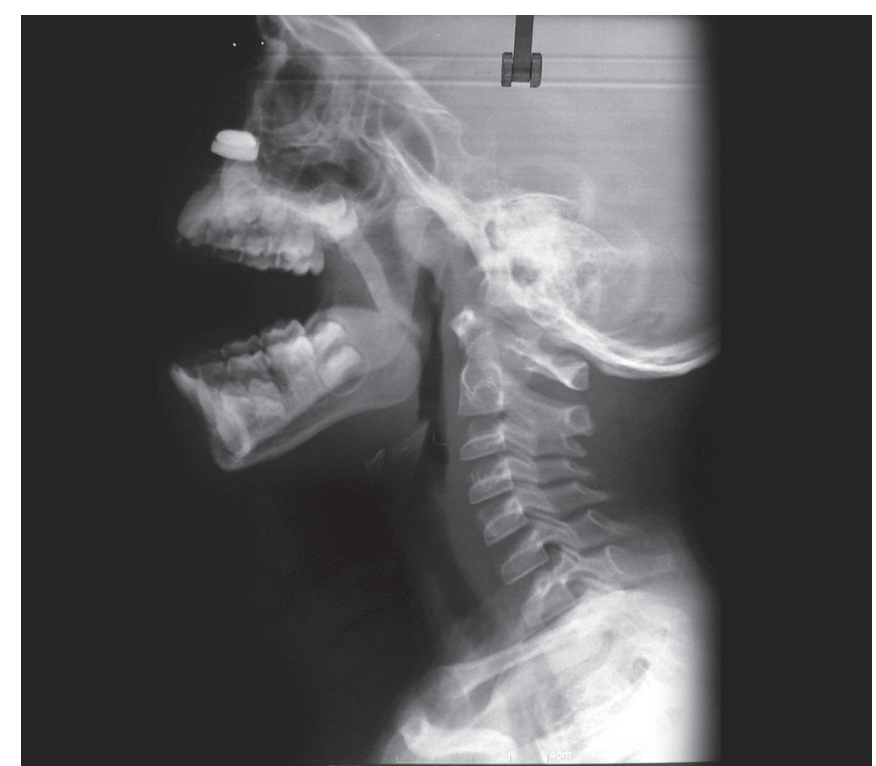

Fig. 1: X-ray nasopharynx: Lateral view showing the foreign body 


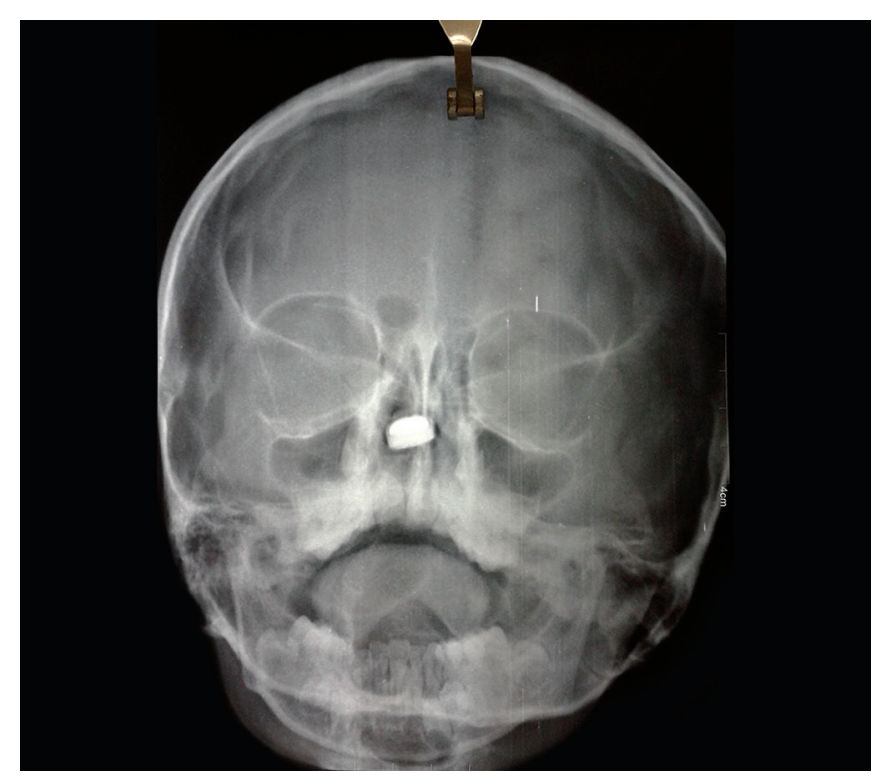

Fig. 2: X-ray paranasal sinus: Water's view showing the foreign body

It was removed (Fig. 3) after releasing it from the margins of septal perforation. A small ointment-soaked pack was placed, which was removed in the evening. Postoperative period was uneventful.

\section{DISCUSSION}

Foreign bodies in the nasal cavity do not feature largely in otolaryngological literature, yet on occasions they may pose a considerable challenge to both the diagnosis and surgical skills of the otolaryngologist, especially if the foreign bodies are harmful and can cause extensive damage to the surrounding mucosa, such as button batteries.

Button batteries contain mercury, silver, zinc, manganese, cadmium, lithium, sulfur oxide, copper, brass, or steel. These are the components of the anode, cathode, and case containing the battery. Button batteries also contain sodium hydroxide or potassium hydroxide to facilitate the electrochemical reaction through the separator. ${ }^{3}$

The impact of button battery depends on the duration it remains in situ, the site at which the battery is lodged, size of the battery, its power, its age (whether new or old, as older batteries cause lesser damage due to diminished contents), and the possibility of heavy-metal absorption. ${ }^{4}$ Increase in the duration between insertion of button battery in the nose and removal can lead to devastating complications like septal perforation, necrosis of nasal mucosa, nasal synechiae, etc. The most common site of leakage from the button battery is the seal. ${ }^{5}$

Button batteries vary in diameter from 7.9 to $23 \mathrm{~mm}$ and in weight from 1 to $10 \mathrm{gm}$. The diameter of disk batteries is less than $15 \mathrm{~mm}$ in $97 \%$ of cases. The most

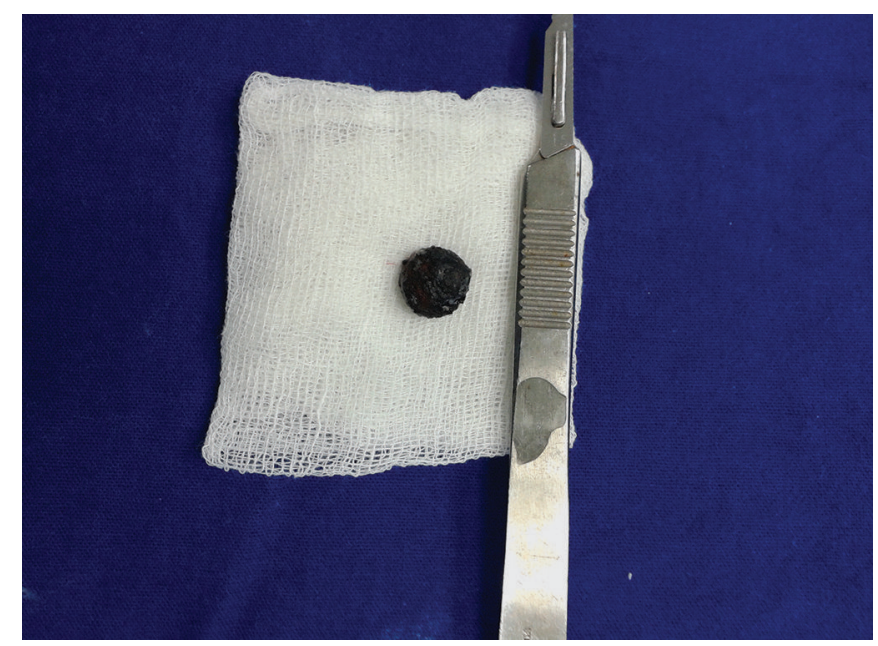

Fig. 3: The battery button removal

frequent sizes are $11.6 \mathrm{~mm}$ in $63 \%$ and $7.9 \mathrm{~mm}$ in $30 \%$ of cases. ${ }^{3}$

In this case, the watch button battery was silver oxide battery with $10.5 \mathrm{~mm}$ in diameter and the color was blackish (normal color is silver) as well as the surrounding mucosa, when it was removed 6 months after being lodged in nasal cavity. Surrounding moisture in the nasal cavity results in corrosion of the battery casing, thereby liberating its contents. More importantly, the batteries can generate local currents resulting in thermal burns and extensive damage to the surrounding mucosa.

Etiology of the tissue damage appears to be threefold: (1) Leakage of the battery contents with direct corrosive damage, (2) direct current effects on the mucosa, and (3) less likely, simple pressure necrosis. Experimental evidence implicates direct current as the primary cause of tissue destruction. The high electrolyte composition of tissue fluids generates a current between the battery cathode and the anode, resulting in the hydrolysis of tissue fluids and the generation of corrosive hydroxides. ${ }^{6,8,10,12}$

Liberation of the contents of the battery causes various types of lesions depending on the localization, with an intense local tissue reaction and liquefaction necrosis. The nasal injuries noted in the literatures included localized nasal mucosal necrosis, septal perforation, facial cellulitis, and lateral nasal wall necrosis. ${ }^{7}$ Nasal button battery impaction may produce mucosal turbinate and septal ulceration in as little as 3 to 6 hours. Necrosis of the inferior turbinate has occurred at 24 hours. ${ }^{8}$ In this case, perforation of the septum was noted. They might be caused by the chemical content of the battery.

Frequently, the foreign body can be seen on anterior rhinoscopy (and sometimes on posterior rhinoscopy), but on several occasions the mucosal edema or granulation will hide it. If the foreign body is easily seen, and the patient is a cooperative child, it is usually possible 
to remove the object through the anterior nares, either without anesthesia, or after spraying local anesthetic solution, such as tetracaine or lidocaine. In this case, the battery was not seen on anterior rhinoscopy and needed X-ray examinations to localize the battery. In the literature, most authors agree with the need for urgent removal of batteries lodged in the nasal cavity. Some authors emphasized that unskilled attempts to remove the foreign body in the emergency department, by personnel without appropriate training, may result in disaster; the foreign body may be displaced backward and may even reach the nasopharynx with risk of inhalation; epistaxis may occur; and a docile child may become terrified and require a general anesthesia and admission to hospital which might have been avoided. ${ }^{9,10}$

In this case, when the patient came to the outpatient department, the visualization of the foreign body in the nasal cavity was already poor. There was foul-smelling discharge in both nasal cavities obstructing the direct view. Therefore, a general anesthesia was scheduled on the next day. However, few recommended immediate removal in the operating room if one cannot retrieve them in the outpatient setting. It is important to wait for ideal facilities, especially an experienced anesthetist. Unskilled manipulation in adverse conditions can lead to inhalation of the foreign body or of blood. At present, there is no guideline as to its removal to prevent occurrence of irreversible complications. ${ }^{10}$

A general anesthetic will be required in the following circumstances: (1) If the patient is uncooperative or very apprehensive; (2) if there is likely to be troublesome bleeding, e.g., if the foreign body is firmly embedded in granulation tissue; (3) if the foreign body is posteriorly placed with a risk of pushing it back into the nasopharynx; (4) if a foreign body is strongly suspected but cannot be found, and more extensive examination of the nose is required, with the opportunity to deal with whatever is found. ${ }^{11}$ In this case, the guidance of imaging system was very helpful and makes the removal easier and could prevent more damage.

There is some evidence that the use of saline or vasoconstrictors may provide electrolytes that increase the necrosis from button batteries. ${ }^{12}$ Thus, when a button battery is suspected to be present in the nose, saline or vasoconstrictors should not be instilled to facilitate the examination until $\mathrm{X}$-rays exclude the existence of button battery foreign body. In this case, the use of adrenaline did not give any bad result, maybe because of the immediate removal of the battery.

\section{CONCLUSION}

Button batteries in the nasal cavity are harmful foreign bodies that can cause tissue damage. Removal of these foreign bodies can be difficult because of poor visualization of the foreign bodies caused by mucosal reaction to the contents of batteries. In this case, the difficulty was caused by profuse bilateral discharge and impaction in the septum.

\section{REFERENCES}

1. Tong MC, Ying SY, van Hasselt CA. Nasal foreign bodies in children. Int J Pediatr Otorhinolaryngol 1996 May;35(3): 207-211.

2. Jatana KR, Litovitz T, Reilly JS, Koltai PJ, Rider G, Jacobs IN. Pediatric button battery injuries: 2013 task force update. Int J Pediatr Otorhinolaryngol 2013 Sep;77(9):1392-1399.

3. Skinner DW, Chui P. The hazards of "button-sized" batteries as foreign bodies in the nose and ear. J Laryngol Otol 1986 Nov;100(11):1315-1318.

4. Thabet MH, Basha WM, Askar S. Button battery foreign bodies in children: Hazards, management, and recommendations. BioMed Res Int 2013;Article ID 846091:1-7.

5. Bhatia R, Singhal SK, Dass A. Button cell causing septal perforation in a child. Clin Rhinol 2010;3(3):161-163.

6. Capo JM, Lucente FE. Alkaline battery foreign bodies of the ear and nose. Arch Otolaryngol Head Neck Surg 1986 May;112(5):562-563.

7. Kalan A, Tariq M. Foreign bodies in the nasal cavities: a comprehensive review of the aetiology, diagnostic pointers, and therapeutic measures. Postgrad Med J 2000 Aug;76(898): 484-487.

8. Tong MCF, Van Hasselt SA, Woo JKS. The hazards of button batteries in the nose. J Otolaryngol 1992 Dec;21(6):458-460.

9. Gomes CC, Sakano E, Lucchezi MC, Porto PR. Button battery as a foreign body in the nasal cavities. Special aspects. Rhinology 1994 Jun;32(2):98-100.

10. Loh WS, Leong JL, Tan HKK. Hazardous foreign bodies: complications and management of button batteries in nose. Ann Otol Rhinol Laryngol 2003 Apr;112(4):379-383.

11. Ransome, J. Foreign bodies in the nose. In: Scott \& Brown's otolaryngology. 5th ed. Butterworths; 1987. p. 276-279.

12. Alvi A, Bereliani A, Zahtz GD. Miniature disc battery in the nose: a dangerous foreign body. Clin Pediatr 1997 Jul;36(7):427-429. 